\title{
Infectious complications from full extension endobronchial ultrasound transbronchial
} needle aspiration

\author{
A.R. Haas
}

ABSTRACT: The development of a convex probe endobronchial ultrasound (CP-EBUS) videobronchoscope to allow real-time transbronchial needle aspiration (TBNA) has been a significant advance in minimally invasive lung cancer staging and diagnosis. Several recent studies have demonstrated CP-EBUS-TBNA to have recovery rivalling that of the current gold standard, cervical mediastinoscopy. These same studies have indicated that the safety of this procedure has no reported complications.

The present case study presents two infectious complications from full extension endobronchial ultrasound transbronchial needle aspiration and discusses possible aetiologies of these infections, as well as implications for future application of this technology.

KEYWORDS: Endobronchial ultrasound, infection, lung cancer, transbronchial needle aspiration

ung cancer staging has evolved significantly over the last 20 yrs. Cervical mediastinoscopy was the traditional approach to mediastinal lymph node evaluation. However, with the advent of flexible transbronchial needle aspiration (TBNA) of lymph nodes, less invasive lung cancer staging became possible. The recent development of a convex probe endobronchial ultrasound (CP-EBUS) videobronchoscope for real-time TBNA visualisation has significantly improved TBNA sensitivity. Utilising this technology, several groups have demonstrated sensitivities of $90-95 \%$, thereby avoiding a significant number of surgical procedures [1,2]. YASUFUKU et al. [3] have also shown that EBUS-TBNA is better than computed tomography (CT) scans and positron emission tomography (PET) scans for the detection of micrometastatic nodal disease. Importantly, this technique has been shown to be very safe, with no reported complications published to date. The present case study describes two patients who developed infectious complications following full extension CP-EBUS-TBNA.

\section{CASE REPORTS}

\section{Patient 1}

A 50-yr-old black American male had an abdominal CT scan performed for vague abdominal complaints. The CT scan was normal with the exception of a $1.5 \times 2.3-\mathrm{cm}$ subcarinal lymph node present on the most cranial cross-sectional images. A chest CT scan performed 4 months later showed the subcarinal lymph node to be $2.5 \times 3.2 \mathrm{~cm}$. The patient was referred for evaluation. He had no cardiopulmonary symptoms and a 35 pack-yr smoking history. His physical examination demonstrated normal vital signs and a medical examination showed no lymphadenopathy or cardiopulmonary abnormalities.

Diagnostic bronchoscopy revealed normal endobronchial anatomy. A large, homogeneous subcarinal mass was identified using CP-EBUS (BFUC160F-OL8; Olympus America Inc., Center Valley, PA, USA) and real-time EBUS-TBNA was performed with the EBUS needle (NA201SX-4022; Olympus America Inc.). Rapid onsite cytology (ROSE) revealed an epithelial malignancy and the cytopathologist requested further tissue for immunohistochemical analysis. Therefore, two further EBUS-TBNA specimens were obtained with the needle extended to its full depth of $3.6 \mathrm{~cm}$ (fig. 1). A substantial quantity of additional specimen was obtained. The patient tolerated the procedure well and was discharged the same day. The cytological-cell block analysis with immunohistochemical stains identified an adenocarcinoma of unknown primary origin.

The patient complained of low-grade fevers and chills $26 \mathrm{~h}$ later, which persisted for another $24 \mathrm{~h}$. A chest radiograph was normal and the patient's symptoms abated over the next $24 \mathrm{~h}$ without
AFFILIATIONS

Interventional Pulmonary Program, Division of Pulmonary Medicine and Critical Care, Jefferson Medical College of Thomas Jefferson University, Philadelphia, PA, USA.

CORRESPONDENCE

A.R. Haas

Section of Interventional

Pulmonology and Thoracic Oncotogy

Division of Pulmonary,

Allergy and Critical Care

University of Pennsylvnia Medical School

823 West Gates Building

3400 Spruce Street

Philadelphia

PA 19104

USA

Fax: 12156140869

E-mail: arhaas@uphs.upenn.edu

Received:

February 182008

Accepted after revision:

October 022008

STATEMENT OF INTEREST

None declared.

European Respiratory Journal Print ISSN 0903-1936 Online ISSN 1399-3003 
intervention. The patient presented to the emergency department 19 days after the bronchoscopy with complaints of epigastric discomfort, dyspnoea and palpitations. A chest radiograph was normal but low voltage was evident on an electrocardiogram. An echocardiogram demonstrated a moderate pericardial effusion with tamponade physiology. Emergent pericardiocentesis resulted in a cloudy yellow fluid which grew Actinomyces odontolyticus and Streptococcus mutans. Following pericardial fluid drainage and several weeks of antimicrobial therapy, the patient recovered completely.

\section{Patient 2}

A 58-yr-old Caucasian female was diagnosed with a 2-mm thick melanoma of the left eyelid. A staging thoracoabdominal CT scan demonstrated a $5.1 \times 4.0-\mathrm{cm}$ mass in the right lower lobe. The patient was referred for evaluation. She had no cardiopulmonary symptoms and a 40 pack-yr smoking history. Her physical examination demonstrated normal vital signs and a medical examination showed no lymphadenopathy or cardiopulmonary abnormalities.

Diagnostic bronchoscopy demonstrated extrinsic compression of the posterior-lateral wall of the bronchus intermedius. Although no paratracheal or subcarinal lymph nodes were visualised by $\mathrm{CP}$-EBUS, a large homogeneous mass was seen posterior to the bronchus intermedius. Real-time EBUS-TBNA of the mass revealed large malignant cells by ROSE. The cytopathologist requested further tissue for immunohistochemical analysis. Consequently, two further EBUS-TBNA specimens were obtained with the needle extended to its full depth of $3.6 \mathrm{~cm}$.
A substantial quantity of additional specimen was obtained. The patient tolerated the procedure well and was discharged the same day. The cytological-cell block analysis with immunohistochemical stains identified a primary squamous cell carcinoma of the lung, not metastatic melanoma.

The patient developed low-grade fevers and a new productive cough with trace haemoptysis $\sim 48 \mathrm{~h}$ later. A 5-day course of azithromycin was prescribed with symptom resolution. Productive cough and low-grade fevers returned 2 days later. At this time, a previously scheduled PET-CT scan was performed and demonstrated not only significant hypermetabolic activity in the primary mass but also that the mass was significantly larger with multiple air pockets (fig. 2). A new hypermetabolic right paratracheal lymph node was present $(1.1 \mathrm{~cm}$ in shortest dimension). Tumour bed contamination and infection by EBUS-TBNA was clinically diagnosed and a prolonged antibiotic course was initiated with full recovery.

\section{DISCUSSION}

Conventional TBNA has been performed for $\sim 20$ yrs and there are exceedingly rare case reports documenting complications. Haemomediastinum following aortic puncture [4, 5], pneumothorax [6], pneumomediastinum, chylothorax [7] and purulent polymicrobial pericarditis [8] have been reported. When compared to conventional TBNA, it would seem intuitive that complications should be minimised with CP-EBUS-TBNA as needle aspiration occurs under real-time visualisation. In the studies published to date for CP-EBUS-TBNA, there have been no complications described.
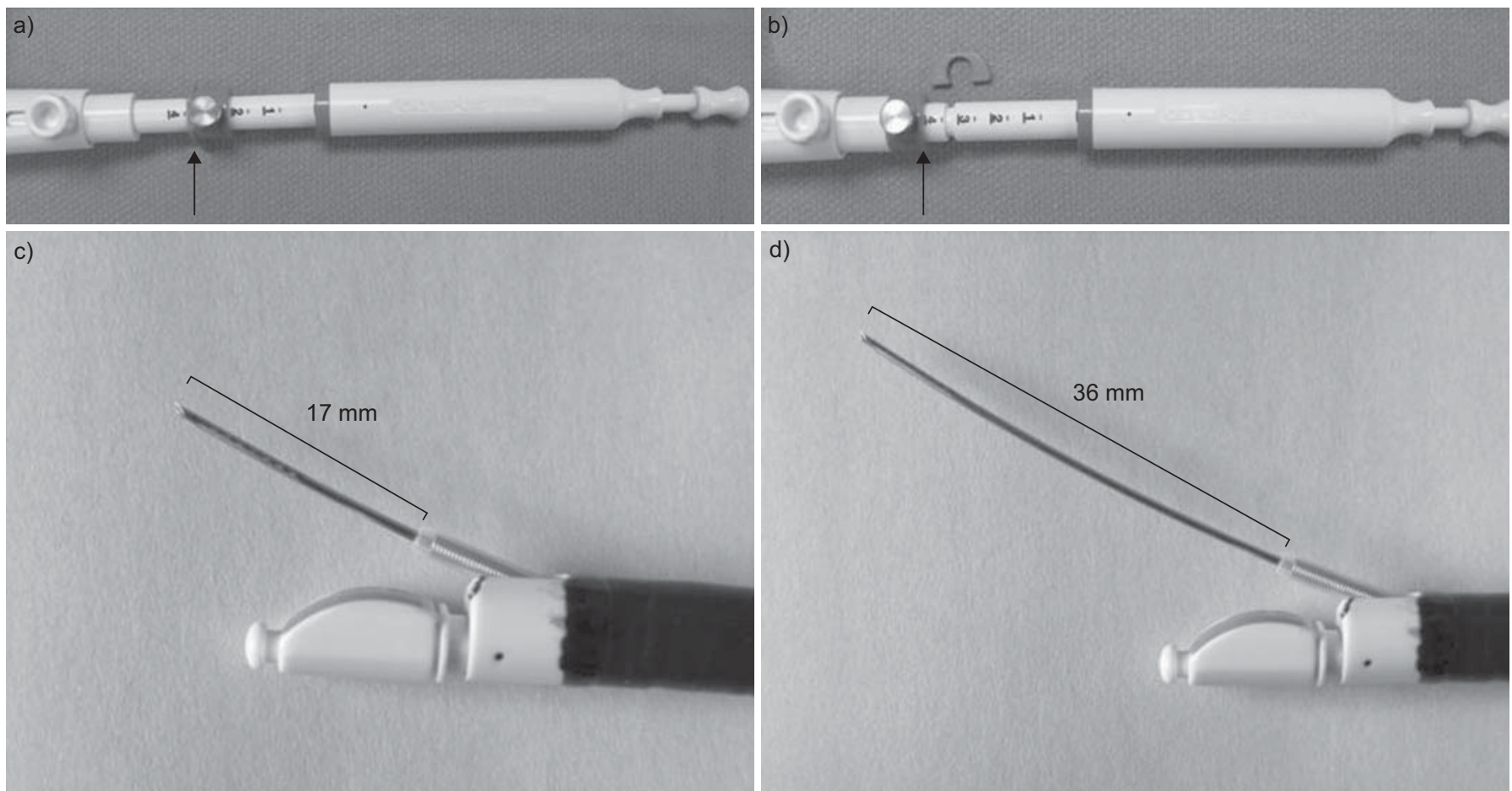

d)

FIGURE 1. The convex probe endobronchial ultrasound needle (NA-201SX-4022; Olympus America Inc., Center Valley, PA, USA) at different depths of penetration. The needle design allows for the operator to determine needle penetration depth. a) The needle contains an adjustable safety (arrow) that allows for the operator to position the needle at a desired depth. b) The adjustable safety (arrow) can be fully extended so the needle can have full penetration depth. c) With the adjustable safety set at $2.5 \mathrm{~cm}$, the needle advances $\sim 17 \mathrm{~mm}$ from the end of the sheath. d) With the adjustable safety positioned to allow full depth, the needle advances $\sim 36 \mathrm{~mm}$ from the end of the sheath, allowing for much deeper tissue penetration. 

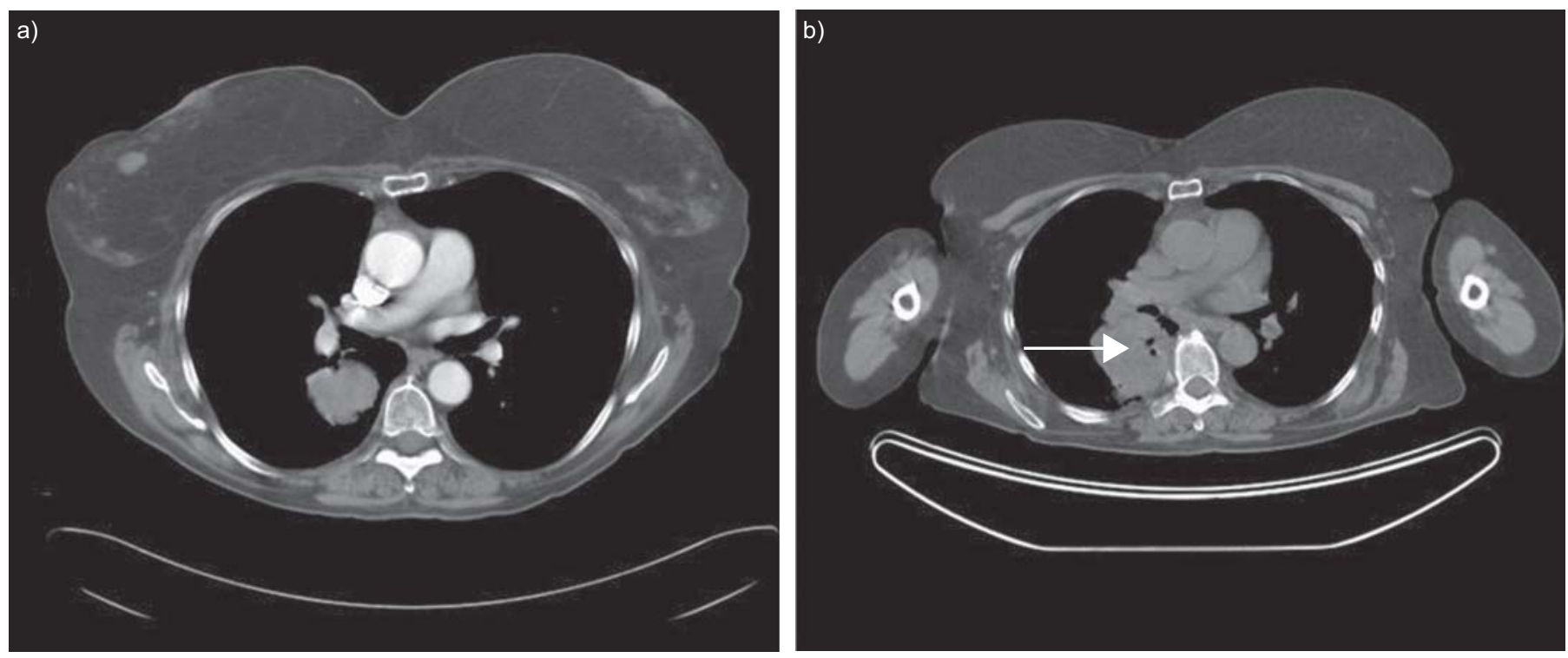

FIGURE 2. Computed tomography (CT) images from patient 2 before and after endobronchial ultrasound transbronchial needle lymph node aspiration (EBUS-TBNA).

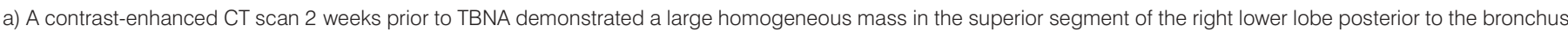

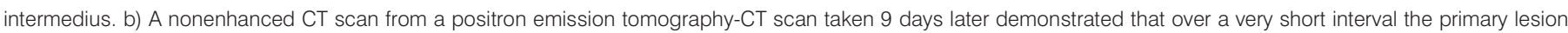
significantly increased in size (from $3.4 \times 2.9 \mathrm{~cm}$ to $6.5 \times 5.3 \mathrm{~cm}$ ), but more importantly now contained multiple small gas bubbles at different levels (arrow)

The present case study reports two patients in whom the development of post-procedure infectious complications appeared to be caused by full-needle extension EBUS-TBNA. Patient 1 developed an initial infectious clinical picture with lowgrade fevers and chills shortly after EBUS-TBNA. These symptoms resolved but were followed within 2 weeks by the development of infectious pericarditis with two different oral organisms. Since the fevers commenced shortly after EBUSTBNA, pericardial space contamination, is likely to have occurred during the procedure. Full-needle extension must have allowed the needle tip to enter the pericardial space to allow for pericardial contamination as the needle tip can be difficult to visualise in all planes when fully extended. Pericardial space needle penetration was not visualised directly during real-time aspiration but must have been the cause of contamination as: 1) the patient never developed mediastinitis which could have spread by continuity into the pericardial space; 2) it would be unlikely for transient bacteraemia to manifest as only infectious pericarditis; and 3) pericardial fluid grew two oral organisms.

Similarly, patient 2 developed a clinical infectious picture shortly after full-needle extension EBUS-TBNA. Although no positive culture data confirmed infection, several factors strongly support EBUS-TBNA mediated contamination of the tumour bed, including: 1) temporal infectious presentation following EBUS-TBNA; 2) rapid expansion of the lung mass containing air pockets 9 days after EBUS-TBNA; 3) the development of new reactive right paratracheal lymphadenopathy; and 4) excellent antimicrobial response. Moreover, it would be unlikely for tumour necrosis, cavitation and secondary infection to occur so rapidly without the initiation of systemic chemotherapy or radiation therapy.

Previous studies with conventional TBNA have investigated the possibility of post-procedure bacteraemia. WITTE et al. [9] performed blood cultures on 47 consecutive patients, 5 and $30 \mathrm{~min}$ after final TBNA and in any patient who developed post-procedure fever within $24 \mathrm{~h}$. No positive blood cultures were obtained in any patient [9]. Interestingly, one case report documented Streptococcus viridans bacteraemia when the patient became febrile $6 \mathrm{~h}$ after TBNA without clinical consequence [10]. In contrast, in both cases presented herein, the infections were unlikely to have been caused by systemic bacteraemia but rather by deposition of oral contaminants into the pericardial space or tumour bed. In support of this potential aetiology, EPSTEIN et al. [8] performed quantitative cultures on seven consecutive patient TBNA samples and discovered polymicrobial aerobic and anaerobic growth in all seven samples. They postulated that as the bronchoscope traversed the naso-oropharyngeal region, the working channel became contaminated. As a result, when the transbronchial needle passed through the working channel, it became contaminated and could potentially inoculate sampled tissue.

A similar mechanism may explain the contamination in the cases presented herein. Although the needle designed for this scope has an outer sheath to minimise sample contamination, this sheath still passed through the working channel. When the needle was deployed at the site of interest, it passed through the distal end of this sheath and must have become contaminated. In addition, needle penetration depth may have contributed to contamination and infection. Furthermore, since larger lesions may have areas of central necrosis, blood flow through these areas could be compromised thereby reducing bacterial inoculation clearance.

Could these infectious complications be due to inadequate bronchoscope processing or operator inexperience? While there are well-documented failures in bronchoscope manufacturing and cleaning procedures that have led to procedure-related infection outbreaks [11-13], this source is unlikely in the present case study for several reasons. First, this technology was new to the author's bronchoscopy programme and the manufacturer 
provided several supervised training sessions on appropriate bronchoscope cleaning, disinfection, storage and utilisation. Secondly, these infections occurred in only two out of $\sim 85$ procedures and were not sequential in nature, suggesting a consistent, prospective problem with bronchoscope cleaning. Finally, the organisms isolated in patient 1 were typical oral organisms, suggesting a contamination method, as described previously.

Operator inexperience with this new technology certainly could have contributed to the infectious pericarditis in patient 1. With full-needle extension, the needle tip can be difficult to visualise as small changes in the ultrasound angle can result in the needle tip being out of plane with the ultrasound; thus the pericardium could be violated but not visualised. In addition, pericardial sac EBUS identification by experienced operators can be difficult, which would be magnified for an inexperienced operator. Novice operator zeal to obtain diagnostic tissue under direct EBUS vision may create a sense of aggressive depth penetrations that could put patients at risk.

Naturally, the question arises as to whether full-needle extension was necessary. The EBUS-TBNA needle design allows for adjustable penetration depth based on visualised lymph node position and size. No CP-EBUS-TBNA published literature describes either the needle depth utilised in the present study, or whether deeper needle penetration improves the yield of diagnostic material obtained. It has been the experience of the present author that if direct EBUS lymph node visualisation could safely accommodate deeper needle passes, larger specimens were acquired. Therefore, to optimise tissue quantity for immunostain analysis in these two cases, full extension EBUSTBNA was performed as the nodal tissue appeared to safely accommodate this depth.

The present case reports bring an important issue to the fore. Several recent studies have demonstrated that expression of certain genes is associated with response to cisplatin-based therapy and survival [14-16]. Moreover, by utilising transbronchial needle lymph node aspiration samples, several groups have shown that evaluation of the epidermal growth factor receptor status can be performed [17-20]. Therefore, with adjustable-depth endobronchial ultrasound needle transbronchial needle lymph node aspiration, the potential may exist for greater tissue acquisition to be used for molecular and genetic analysis to guide therapy in unresectable patients, but at the potential cost of increased infection risk. Importantly, novice operators should exercise appropriate restraint to ensure needle depth is appropriately determined so vital structures are not unintentionally violated, leading to complications.

\section{REFERENCES}

1 Yasufuku K, Chiyo M, Koh E, et al. Endobronchial ultrasound guided transbronchial needle aspiration for staging of lung cancer. Lung Cancer 2005; 50: 347-354.

2 Herth FJ, Ernst A, Eberhardt R, Vilmann P, Dienemann H, Krasnik M. Endobronchial ultrasound-guided transbronchial needle aspiration of lymph nodes in the radiologically normal mediastinum. Eur Respir J 2006; 28: 910-914.

3 Yasufuku K, Nakajima T, Motoori K, et al. Comparison of endobronchial ultrasound, positron emission tomography, and CT for lymph node staging of lung cancer. Chest 2006; 130: 710-718.
4 Kucera RF, Wolfe GK, Perry ME. Hemomediastinum after transbronchial needle aspiration. Chest 1986; 90: 466.

5 Agli LL, Trisolini R, Burzi M, Patelli M. Mediastinal hematoma following transbronchial needle aspiration. Chest 2002; 122: 1106-1107.

6 Wang KP, Brower R, Haponik EF, Siegelman S. Flexible transbronchial needle aspiration for staging of bronchogenic carcinoma. Chest 1983; 84: 571-576.

7 Mobeireek AF, Quraishi E, Zeitouni M, Al-Dalaan A. Chylothorax complicating transbronchial needle aspiration: a case report. J Bronchol 2007; 14: 127-128.

8 Epstein SK, Winslow CJ, Brecher SM, Faling LJ. Polymicrobial bacterial pericarditis after transbronchial needle aspiration. Case report with an investigation on the risk of bacterial contamination during fiberoptic bronchoscopy. Am Rev Respir Dis 1992; 146: 523-525.

9 Witte MC, Opal SM, Gilbert JG, et al. Incidence of fever and bacteremia following transbronchial needle aspiration. Chest 1986; 89: 85-87.

10 Watts WJ, Green RA. Bacteremia following transbronchial fine needle aspiration. Chest 1984; 85: 295.

11 Kirschke DL, Jones TF, Craig AS, et al. Pseudomonas aeruginosa and Serratia marcescens contamination associated with a manufacturing defect in bronchoscopes. $N$ Engl J Med 2003; 348: 214-220.

12 Ramsey AH, Oemig TV, Davis JP, Massey JP, Torok TJ. An outbreak of bronchoscopy-related Mycobacterium tuberculosis infections due to lack of bronchoscope leak testing. Chest 2002; 121: 976-981.

13 Wilson SJ, Everts RJ, Kirkland KB, Sexton DJ. A pseudooutbreak of Aureobasidium species lower respiratory tract infections caused by reuse of single-use stopcocks during bronchoscopy. Infect Control Hosp Epidemiol 2000; 21: 470-472.

14 Chen HY, Yu SL, Chen $\mathrm{CH}$, et al. A five-gene signature and clinical outcome in non-small-cell lung cancer. $N$ Engl J Med 2007; 356: 11-20.

15 Zheng Z, Chen T, Li X, Haura E, Sharma A, Bepler G. DNA synthesis and repair genes RRM1 and ERCC1 in lung cancer. N Engl J Med 2007; 356: 800-808.

16 Olaussen KA, Dunant A, Fouret P, et al. DNA repair by ERCC1 in non-small-cell lung cancer and cisplatin-based adjuvant chemotherapy. N Engl J Med 2006; 355: 983-991.

17 Horiike A, Kimura H, Nishio $\mathrm{K}$, et al. Detection of epidermal growth factor receptor mutation in transbronchial needle aspirates of non-small cell lung cancer. Chest 2007; 131: 1628-1634.

18 Nakajima T, Yasufuku K, Suzuki M, et al. Assessment of epidermal growth factor receptor mutation by endobronchial ultrasound-guided transbronchial needle aspiration. Chest 2007; 132: 597-602.

19 Shih JY, Gow CH, Yu CJ, et al. Epidermal growth factor receptor mutations in needle biopsy/aspiration samples predict response to gefitinib therapy and survival of patients with advanced nonsmall cell lung cancer. Int $J$ Cancer 2006; 118: 963-969.

20 Lim EH, Zhang SL, Yu K, et al. An alternative approach to determining therapeutic choices in advanced non-small cell lung carcinoma (NSCLC): maximizing the diagnostic procedure and the use of low-volume lung biopsies. J Thorac Oncol 2007; 2: 387-396. 